\author{
Military Technical College \\ Kobry El-Kobbah, \\ Cairo, Egypt.
}

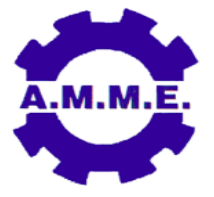

\title{
DYNAMIC SIMULATION OF TANK GUN RECOIL CYCLE
}

\author{
W. M. El-Saady*, A. A. Abd Allah**, A. Z. Ibrahim and A. H. Hussien*
}

\begin{abstract}
A mathematical model is developed - using MATLAB Simulink software package - to simulate, predict, and evaluate the performance of gun recoil system. The used gun is a tank gun with a concentric recoil mechanism. The results are presented in this paper can provide a clear understanding of design parameters affecting the behavior of recoil mechanism and/or improving the performance of recoil mechanisms in the future.
\end{abstract}

A complete study of the constructional parameters that affect the performance of the concentric recoil system is carried out. Then, a mathematical model that includes all governing equations of recoil cycle is developed. Finally, the model is tested for a case study and the results is recorded and compared with others resulting from actual firing test.

This paper starts with studying the construction of concentric recoil mechanism for tank gun and analysis of the different forces and resistances influencing the recoiling parts during recoil cycle; then, introducing the equation of motion of recoiling parts during recoil cycle and finally, constructing the simulation program to obtain results.

Comparison of simulation results and others obtained from actual firing shows a good match that creates some confidence in the mathematical model. This confidence encourages reliance on this model in evaluation, development, and design of such systems.

\section{KEY WORDS}

Concentric recoil mechanism, recoil system, interior pressure in gun recoil cylinder, gun recoil simulation, gun recoil cycle.

* Egyptian Armed Forces.

** Dept. of Mechanical Eng., Modern Academy, El-Maadi, Cairo. 


\section{NOMENCLATURE}

\begin{tabular}{|c|c|c|c|}
\hline$P_{B}$ & Force of powder gases & $\alpha_{12}$ & Contraction factor of flow 12 \\
\hline$K$ & Hydraulik resistance of recoil brake & $P_{R o}$ & Initial recuperator force \\
\hline$X$ & Recoil distance & $\boldsymbol{k}_{R}$ & Recuperator spring stiffness \\
\hline $\boldsymbol{V}$ & Recoil velocity & $X_{\text {so }}$ & Recuperator precompression \\
\hline$D_{i}$ & Inner diameter of recoil cylinder & $A_{x}$ & Throttling area of liquid flow during recoil \\
\hline$d_{P R}$ & Diameter of piston rod & $A_{P B}$ & Working area of recoil brake during recoil \\
\hline$d_{p}$ & Diameter of piston head & $A_{P B C}$ & Working area of recoil brake in counter recoil \\
\hline$p_{1}$ & Pressure in recoil cylinder during recoil & $\rho_{L}$ & Working liquid density \\
\hline$v_{F 12}$ & Flow velocity from space 1 to space 2 & & Friction and local losses coefficient \\
\hline$m_{R}, Q_{R}$ & Mass, weight of recoiling parts & $K_{\text {spear }}$ & Hydraulic resistance due to spear effect \\
\hline $\boldsymbol{R}_{f}$ & Total friction resistances & $k_{L s 2}$ & Losses coefficient from cavity to space 2 \\
\hline$P_{R}$ & Recuperator force & $\alpha_{s 2}$ & Contraction factor of flow from cavity to 2 \\
\hline$\varphi$ & Angle of elevation & $A_{\text {spear }}$ & Working area of spear \\
\hline$f$ & Coefficient of friction in barrel guides & $A_{x s}$ & Throttling area between cavity and spear \\
\hline $\boldsymbol{v}$ & \multicolumn{3}{|c|}{ Coefficient of friction in recoil system stuffing boxes } \\
\hline
\end{tabular}

\section{INTRODUCTION}

During firing using a gun with recoiling barrel, the gun recoil system, which consists mainly of a recuperator and a recoil brake is responsible of attenuation of the impulse transferred to the recoiling parts due to the driving impulse of powder gases and returns the recoiling parts to their initial position. The recoil brake task is to dissipate a large portion of the kinetic energy of recoiling parts; while the recuperator task is to accumulate a portion of the recoil energy in order to use them for counter recoil.

The recoil brake is simply a working cylinder full of liquid. The relative movement of the piston and cylinder during recoil and counter recoil forces the working liquid to pass through narrow orifices. The viscosity of the working fluid provides the required resistance to recoil motion. By controlling the throttling areas of recoil brake along the recoil length, the hydraulic resistance $K$ and the recoil parameters (recoil distance $X$ and recoil velocity $V$ ) are adjusted for proper design.

The recuperator of the tank gun under investigation is a spring type one. The precompression force of the spring performs work satiesfying complete counter recoil of recoiling parts. The surplus energy is attenuated due to the reversed motion of liquid in recoil brake. Employing an additional cushion near the end of counter recoil is essential for decreasing impact on the front part of recoil system and recoil brake cylinder.

Solving the problem of tank gun recoil cycle may be summarized in four steps:

1) Derivation of the driving impulse affecting the recoiling parts. 
2) Determination of resistances affecting recoil cycle which requires awareness of the constrution of the recoil system.

3) Modeling of the equation of motion of gun recoil cycle using a proper mathematical model to investigate the recoil parameters.

4) Finally verifiyng the obtained results.

\section{TANK GUN RECOIL SYSTEM CONSTRUCTION}

The construction of the concentric recoil system of the tank gun under investigation is shown in Figure 1. The recoil brake piston is fixed with the barrel; the recuperator is assembled between the recoil brake piston head rear face and the rear end of recoil cylinder. When the barrel assembly recoils, the liquid inside the recoil cylinder is forced to pass through the annular area between the recoil brake piston head and the inner surface of recoil cylinder. The front cushioning is a spear and cavity which provides an additional hydraulic resistance during counter recoil.

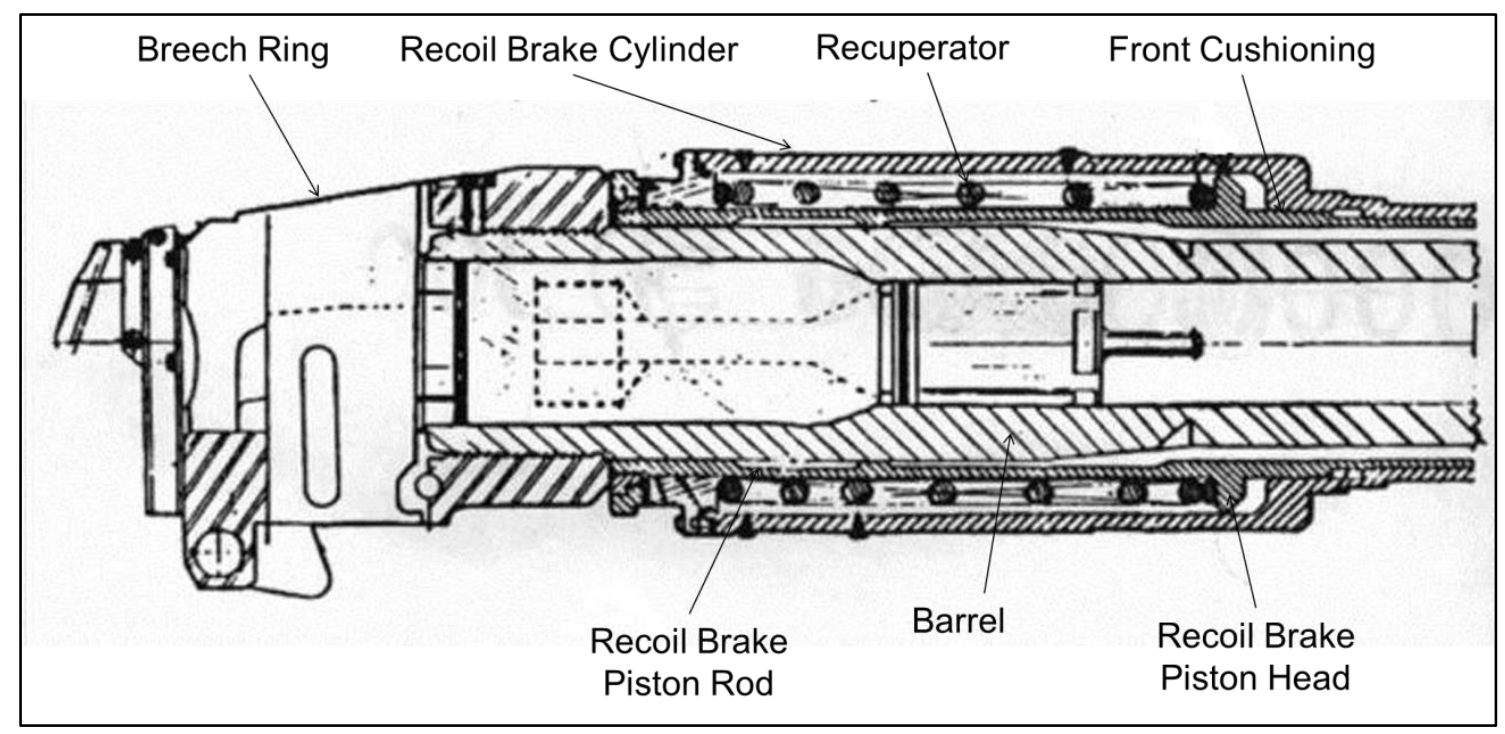

Figure 1. Scheme of tank gun recoil system.

The function during recoil is illustrated in Fig. 2. The hydraulic resistance arises due to pressure in space 1 that affects the projected area of the piston head.

\section{MATHEMATICAL MODEL AND SIMULATION PROGRAM}

The mathematical model - as shown in Figure 3 - consists of the equation of motion of recoiling parts and all equations describing the affecting forces.

The equation of motion of recoiling parts during recoil can be written as:

$$
m_{R} \frac{d^{2} X}{d t^{2}}=m_{R} \frac{d V}{d t}=P_{B}-K-P_{R}-R_{f}+Q_{R} \sin \varphi
$$




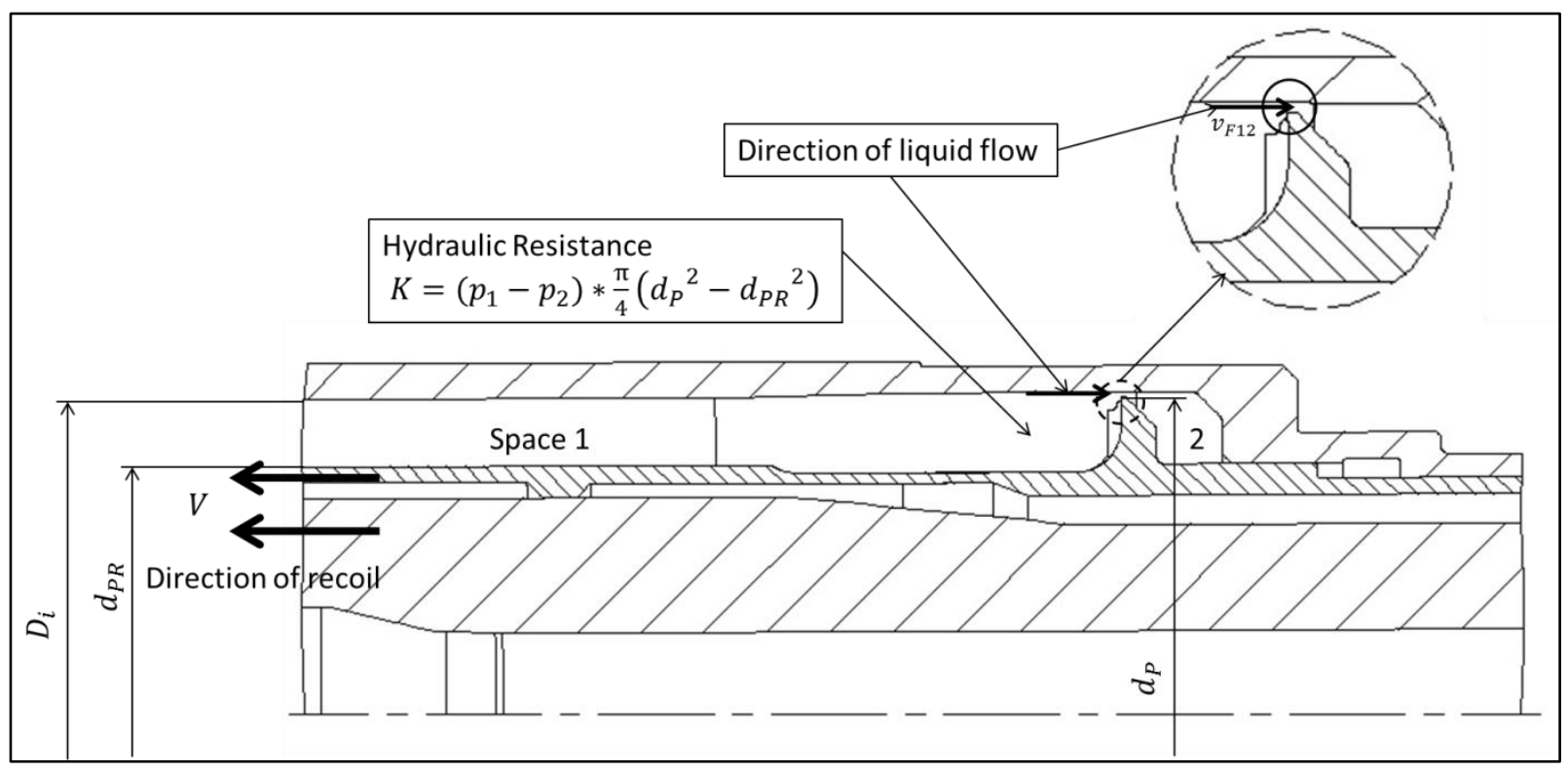

Figure 2. Function during recoil of tank gun recoil system.

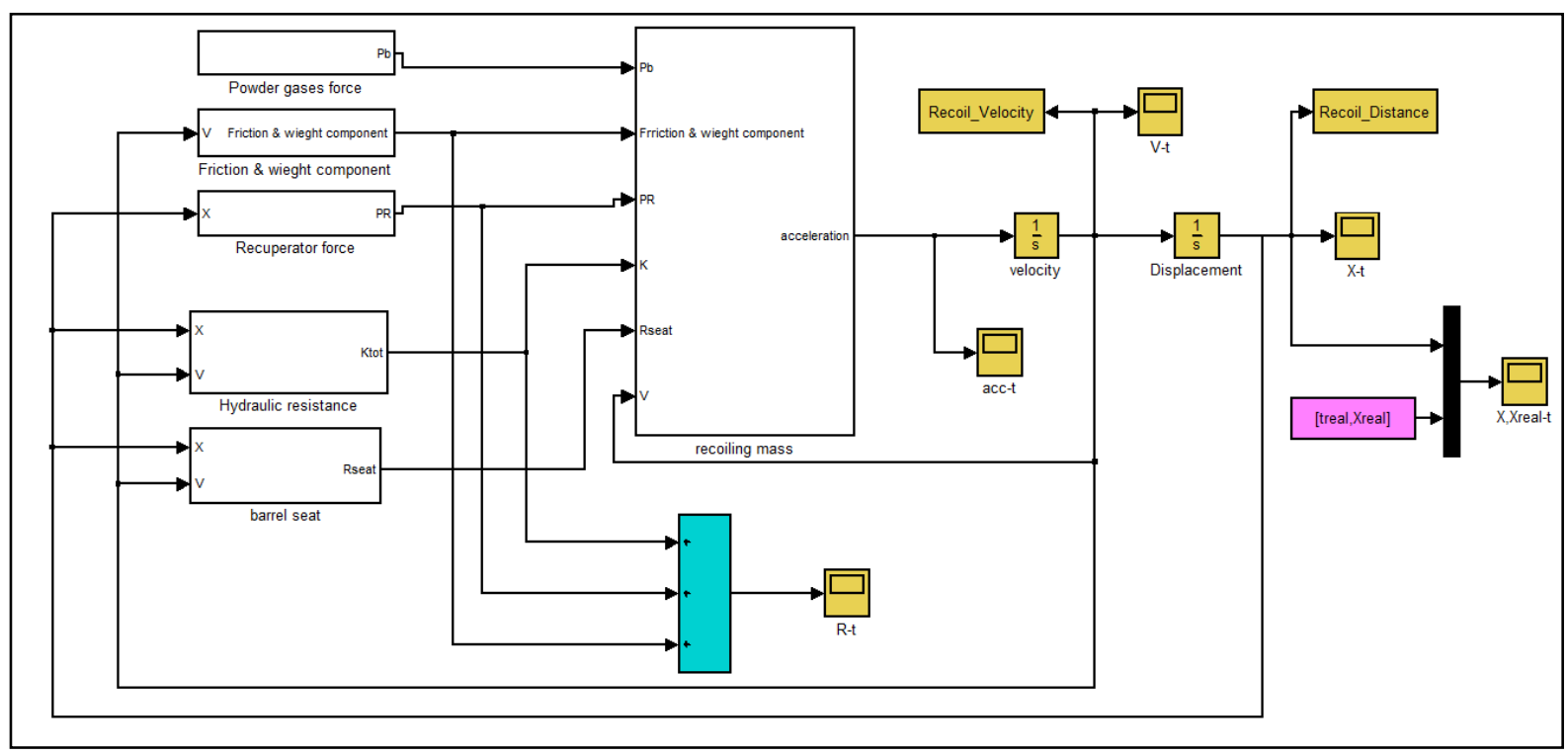

Figure 3. Mathematical model of recoil cycle simulation.

1. The force of powder gases $P_{B}$ is the driving force. It can be determined from the interior ballistic solution which gives the pressure course inside the barrel till the muzzle. The impulse of powder gases during the Additional Action of Powder Gases (AAPG) should be considered to obtain the total driving impulse of the recoiling parts. In this paper, the impulse of powder gases is determined from measurments and set as the input for the simulation program to realise more reliable results for the same input impulse given to the recoiling parts in the mathematical model and experimental work.

The course of force of powder gases is represented in Fig. 4. 


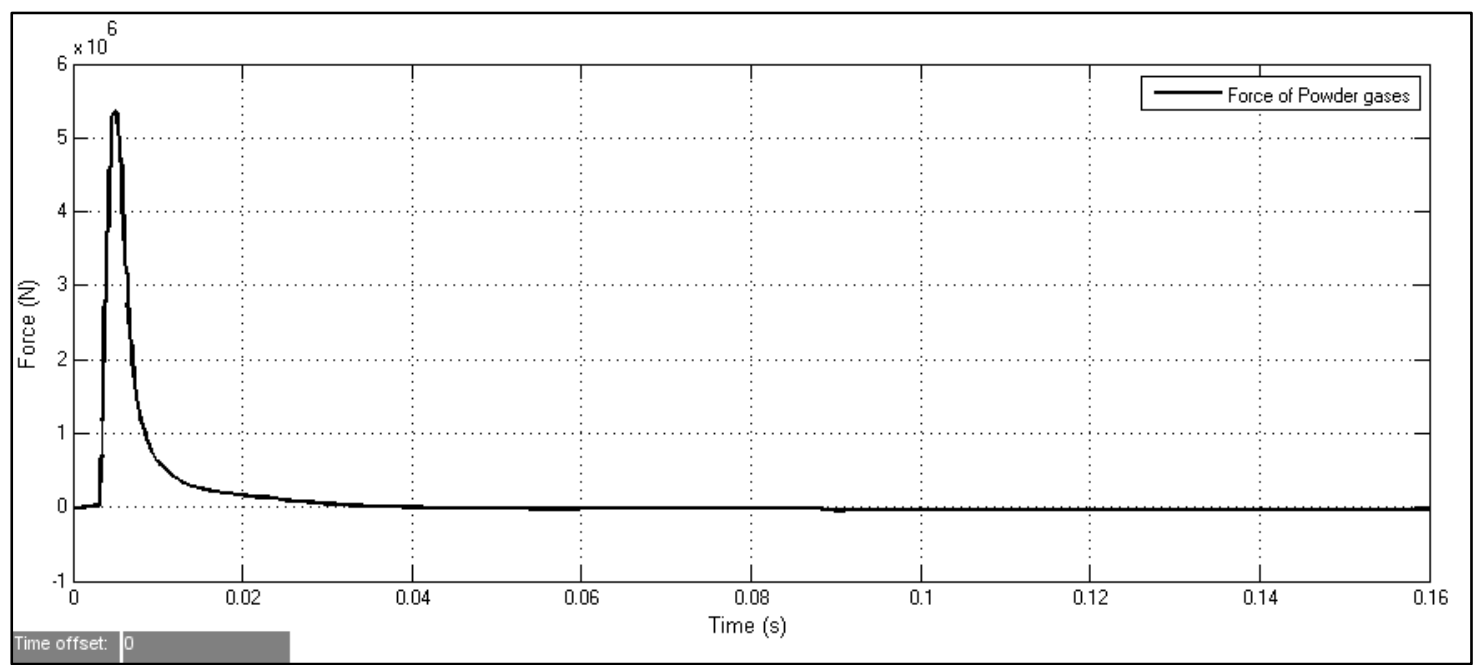

Figure 4. Course of force of powder gases affecting the recoiling parts.

2. The total friction resistances affecting recoil $R_{f}$ is due to the friction in barrel guides besides the friction in stuffing boxes of recoil system.

$$
R_{f}=(f \cos \varphi+v) * Q_{R}
$$

The recoiling mass weight component is a function of the elevation angle $Q_{R} \cdot \sin \varphi$. The friction coeffeicient in stuffing box $v$ is smaller during counter recoil than during recoil as the velocity of counter recoil is much less than the velocity of recoil. The course of friction resistance is assumed to be constant along the recoil distance for a specific elevation angle.

3. The recuperator force along the recoil distance is simply a characteristic curve for a spring depending on the precompression force and the spring stiffness.

So, the course of recuperator force along the recoil distance $X$ is:

$$
P_{R}=k_{R} *\left(X_{s o}+X\right)
$$

4. According to references [1], [2], [3] and [4], the course of hydraulic resistance during recoil cycle is determined by applying continuity and energy equations for the flow inside recoil cylinder to get the course of pressure difference of liquid $\left(p_{1}-p_{2}\right)$.

\section{Continuity equation:}

$$
A_{P B} \cdot V=\alpha_{12} \cdot A_{x} \cdot v_{F 12}
$$

\section{Energy equation:}

It can be reduced to Bernoulli's equation as follows:

Pressure head + Velocity head + Gravity head $=$ Constant

$$
\frac{p_{1}}{\rho_{L} \cdot g}+\frac{V^{2}}{2 g}+y_{1}=\frac{p_{2}}{\rho_{L} \cdot g}+\frac{v_{F 12}^{2}}{2 g}+y_{2}+\Delta p_{\text {losses }}
$$


where,

$\Delta p_{\text {losses }}=\xi_{1} \cdot \frac{\rho_{L} \cdot v_{F 12}^{2}}{2}$ and $\xi_{1}$ is friction and local losses coefficient.

When firing at zero elevation,

$$
p_{1}+\frac{\rho_{L} \cdot V^{2}}{2}=p_{2}+k_{L 12} \frac{\rho_{L} \cdot v_{F 12}{ }^{2}}{2}
$$

where $k_{L 12}=1+\xi_{1}$

Substituting from (4) into (6), the relation of pressure inside space (1) can be represented as follows:

$$
p_{1}-p_{2}=\frac{\rho_{L} \cdot V^{2}}{2}\left[k_{L 12}\left(\frac{A_{P B}}{\alpha_{12} \cdot A_{x}}\right)^{2}\right]
$$

Thus, the hydraulic resistance $K$ can be represented as follows:

$$
K=A_{P B}\left(p_{1}-p_{2}\right)=\frac{\rho_{L} \cdot A_{P B} \cdot V^{2}}{2}\left[k_{L 12}\left(\frac{A_{P B}}{\alpha_{12} \cdot A_{x}}\right)^{2}\right]
$$

And during counter recoil, the process is reversed and the hydraulic resistance will be:

$$
K_{C}=A_{P B C}\left(p_{2}-p_{1}\right)=\frac{\rho_{L} \cdot A_{P B C} \cdot V^{2}}{2}\left[k_{L 21}\left(\frac{A_{P B C}}{\alpha_{21} \cdot A_{x}}\right)^{2}\right]
$$

Applying the same equations on the front cushioning considering that according to construction, the cushion effect happens in the most forward part of counter recoil and decisively, before the end of counter recoil by a distance $X=0.065(\mathrm{~m})$.

\section{Cushion effect:}

The cushion is a spear and cavity principle at the end of counter recoil track as shown in Fig. 5.

Applying continuty and energy equations on the cushion, the hydraulic resistance due to spear effect can be evaluated.

$$
K_{\text {spear }}=p_{2} \cdot A_{\text {spear }}+\frac{\rho_{L} \cdot A_{\text {spear }}}{2}\left[k_{L s 2}\left(\frac{A_{\text {spear }}}{\alpha_{s 2} \cdot A_{x S}}\right)^{2}\right] \cdot V^{2}
$$

where,

$$
A_{x s}=\frac{\pi}{4}\left(d_{\text {cavity }}{ }^{2}-d_{\text {spear }}{ }^{2}\right), \quad A_{\text {spear }}=\frac{\pi}{4}\left(d_{\text {spear }}{ }^{2}-d_{P R C}^{2}\right)
$$

The hydraulic resistance model and the spear effect model are shown in Figure 5 and Figure 6, respectively. 


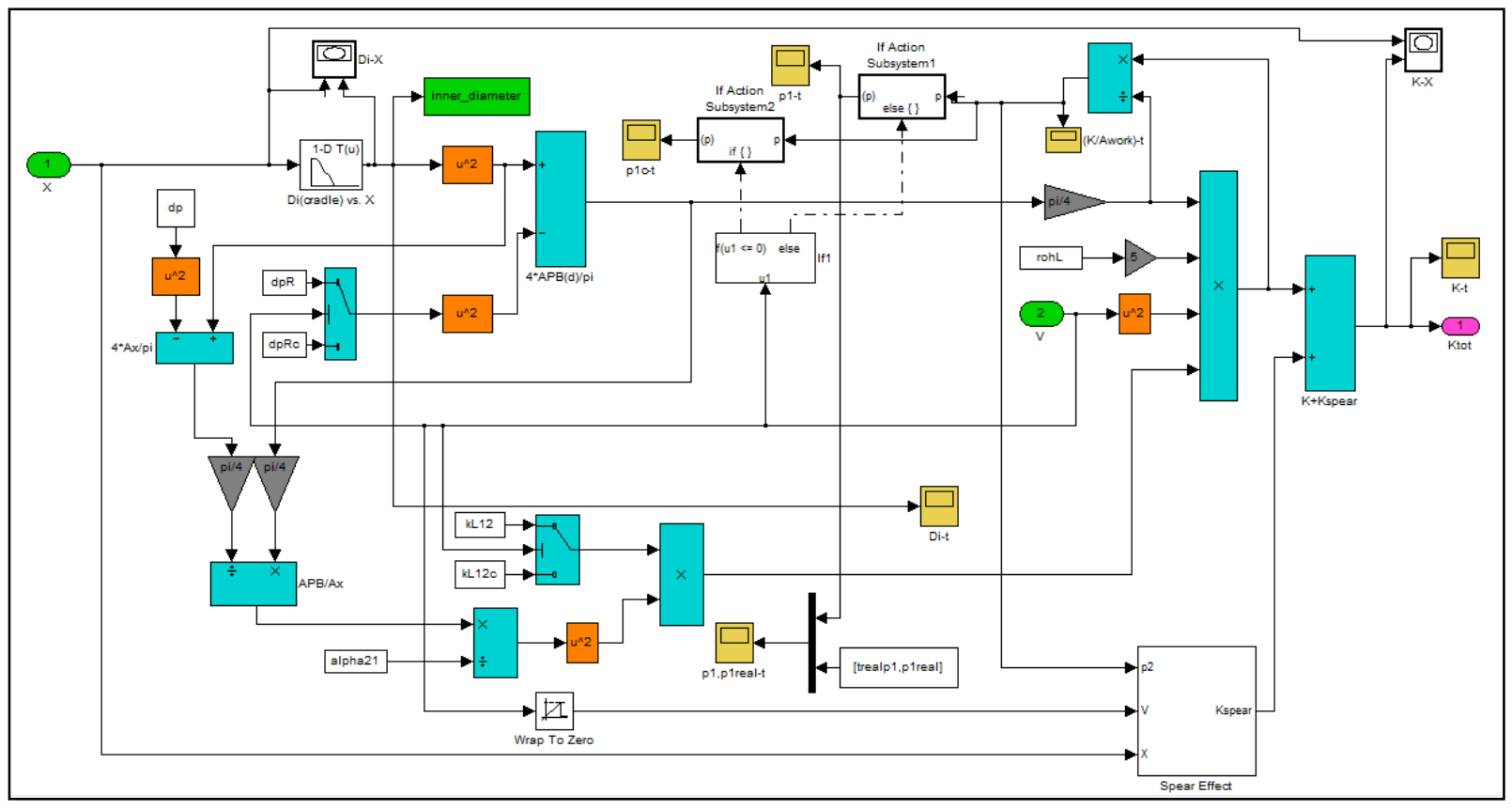

Figure 5. Hydraulic resistance model.

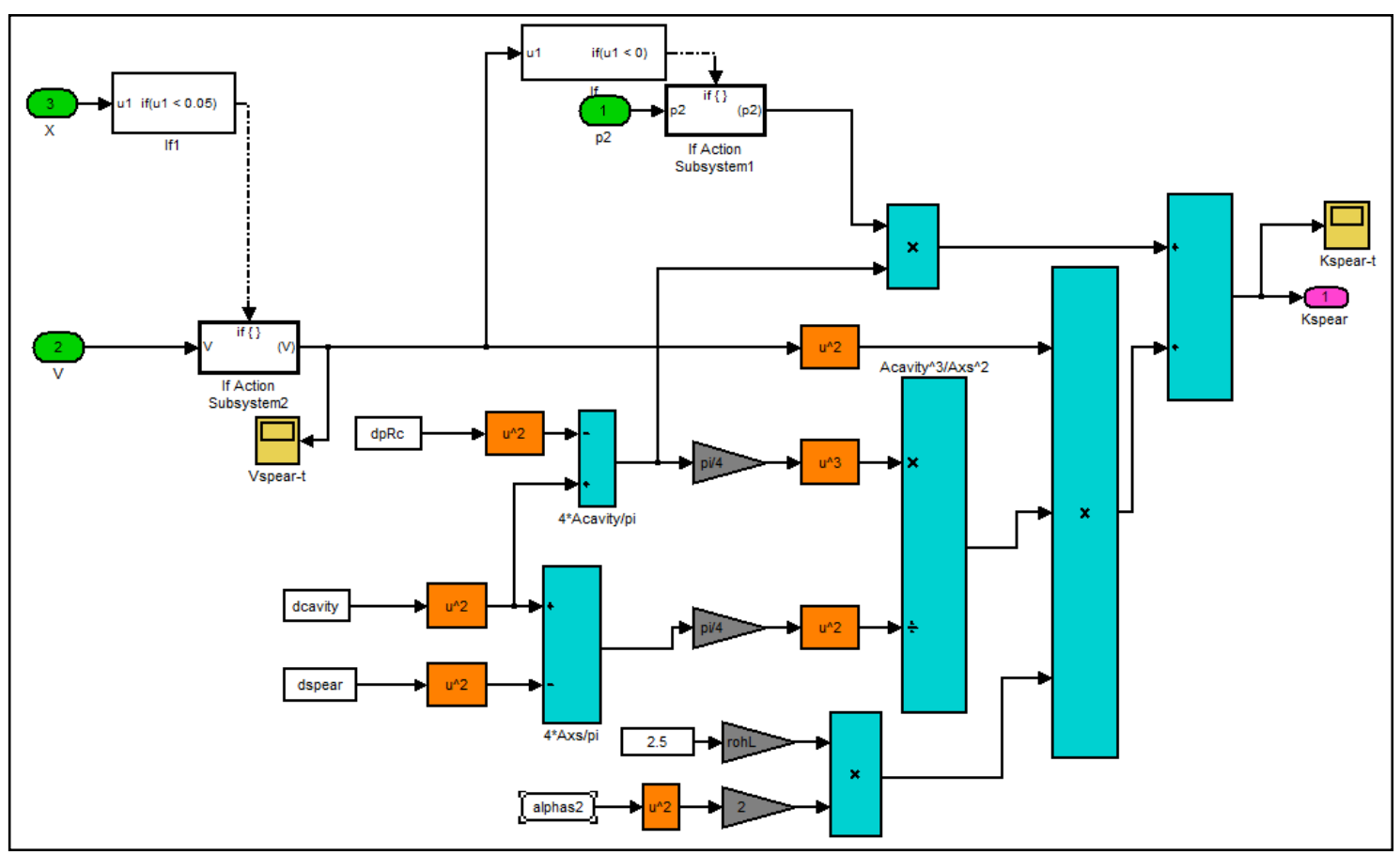

Figure 6. Spear effect model. 


\section{EXPERIMENTAL WORK, RESULTS AND DISCUSSIONS}

In the following, the results of the mathematical model compared with experimental results and discussions for both results are presented.

\section{Experimental Work Description}

The measuring system is shown in Figure 7 and it consists of:

1) Pressure transducer with internal memory placed inside the cartridge case to measure and to record the pressure-time variation inside the barrel.

2) Piezo-electric pressure transducer screwed in recoil brake cylinder body in place of replenisher connection.

3) Amplifier and data acquisition system (DAQ) connected to Lab view measuring software to amplify, digitize and display the signal of the pressure transducer.

4) High speed camera with Phantom software to record the recoil cycle.

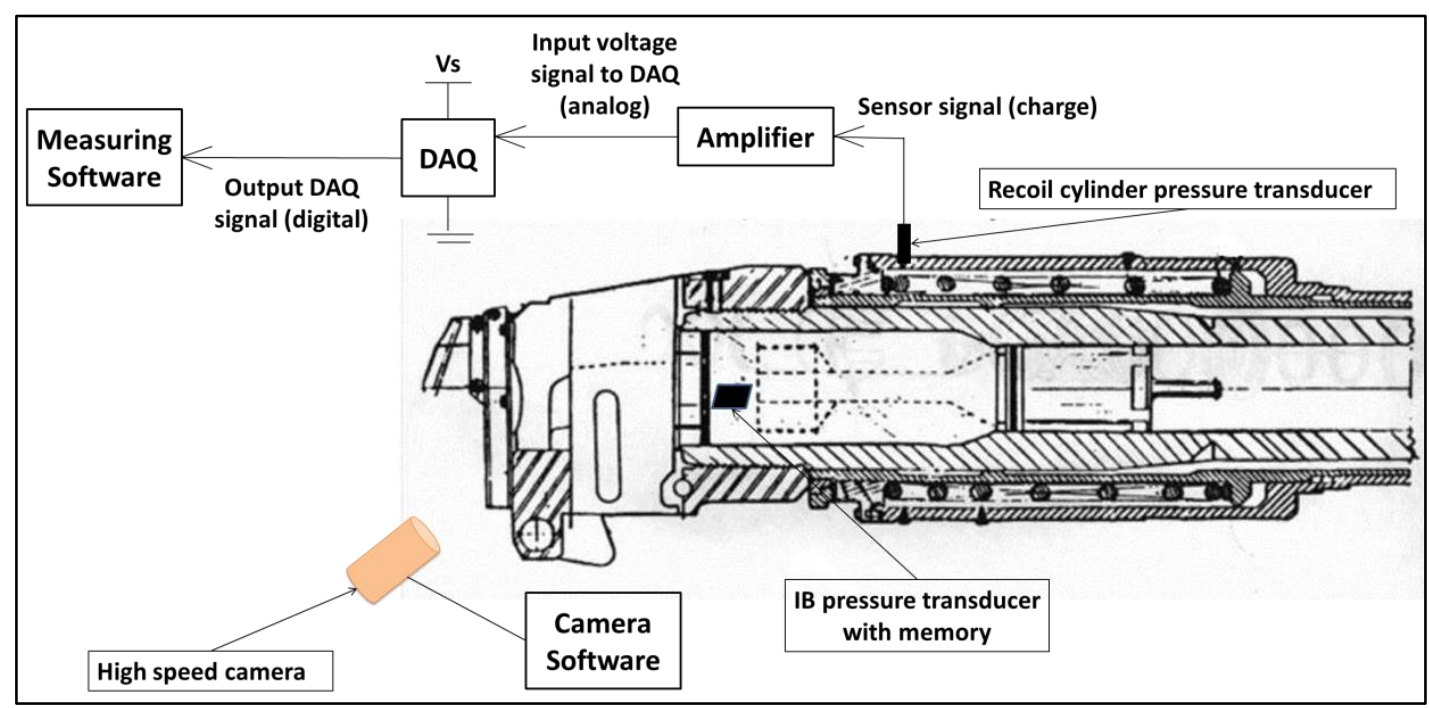

Figure 7. Sheme of measuring system.

\section{Results}

- The force of powder gases course with time is previously shown in Figure 4.

- The simulated and measured recoil distance courses $(X-t)$ are shown in Figure 8.

- The simulated and measured liquid pressures inside recoil brake cylinder during recoil $\left(p_{1}-t\right)$ are shown in Figure 9.

- The velocity of recoiling parts with time during recoil cycle according to simulation is shown in Figure 10. 


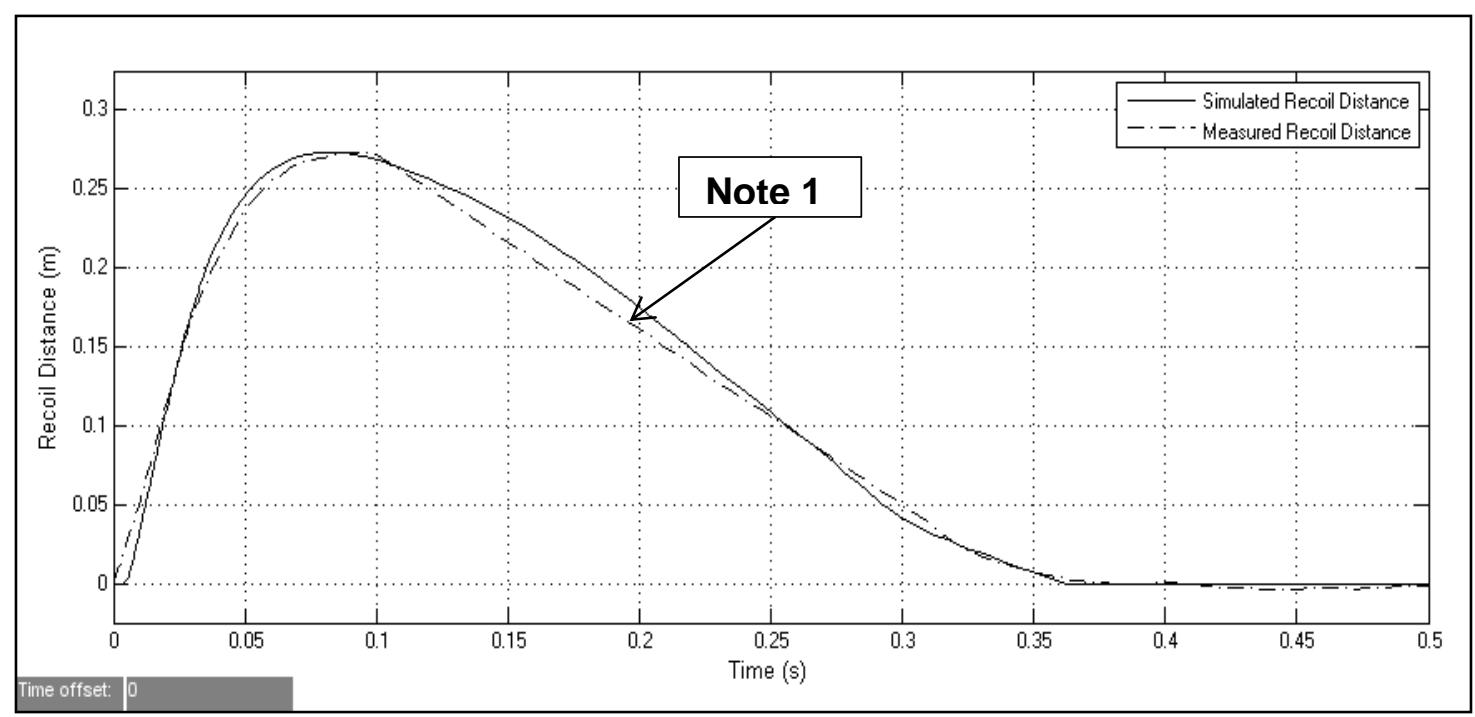

Figure 8. Course of recoil distance.

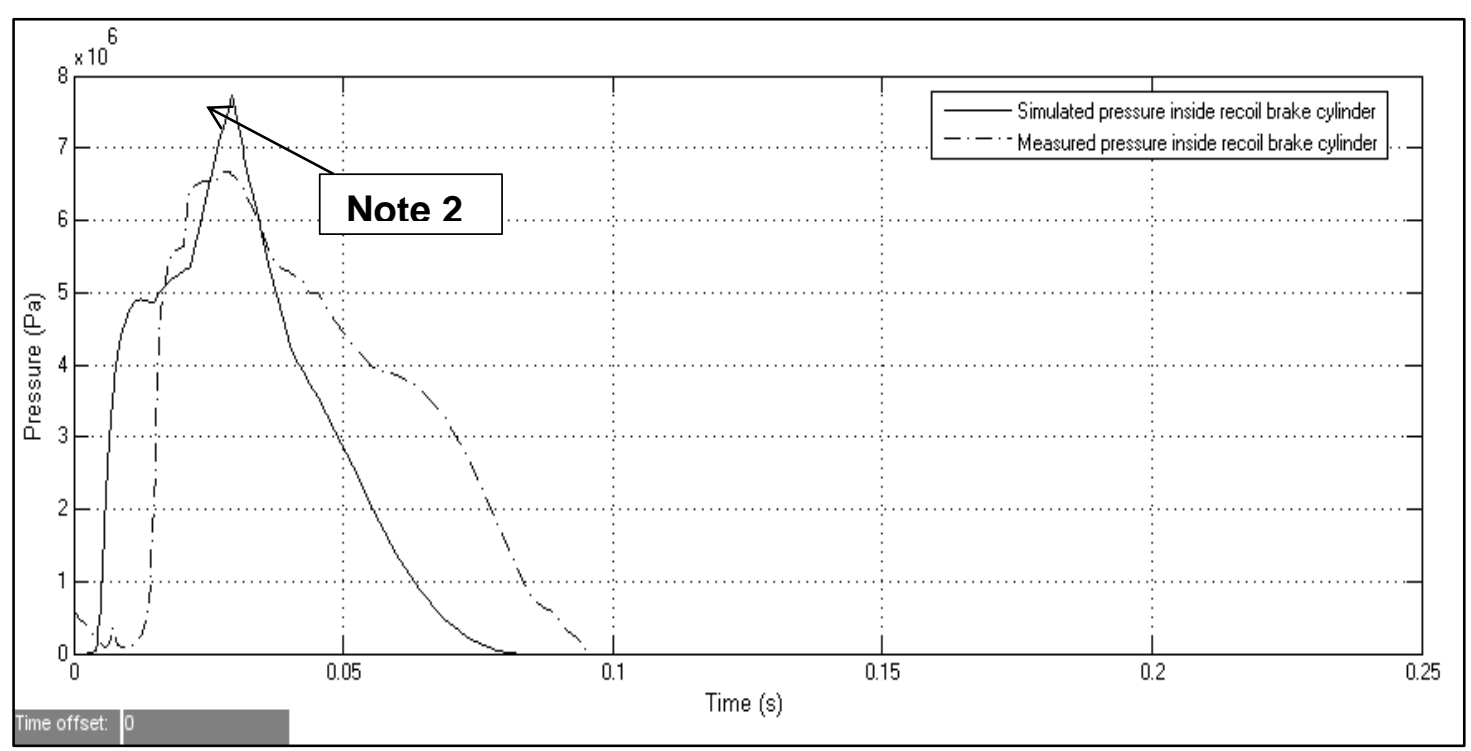

Figure 9. Liquid pressure inside recoil brake cylinder during recoil.

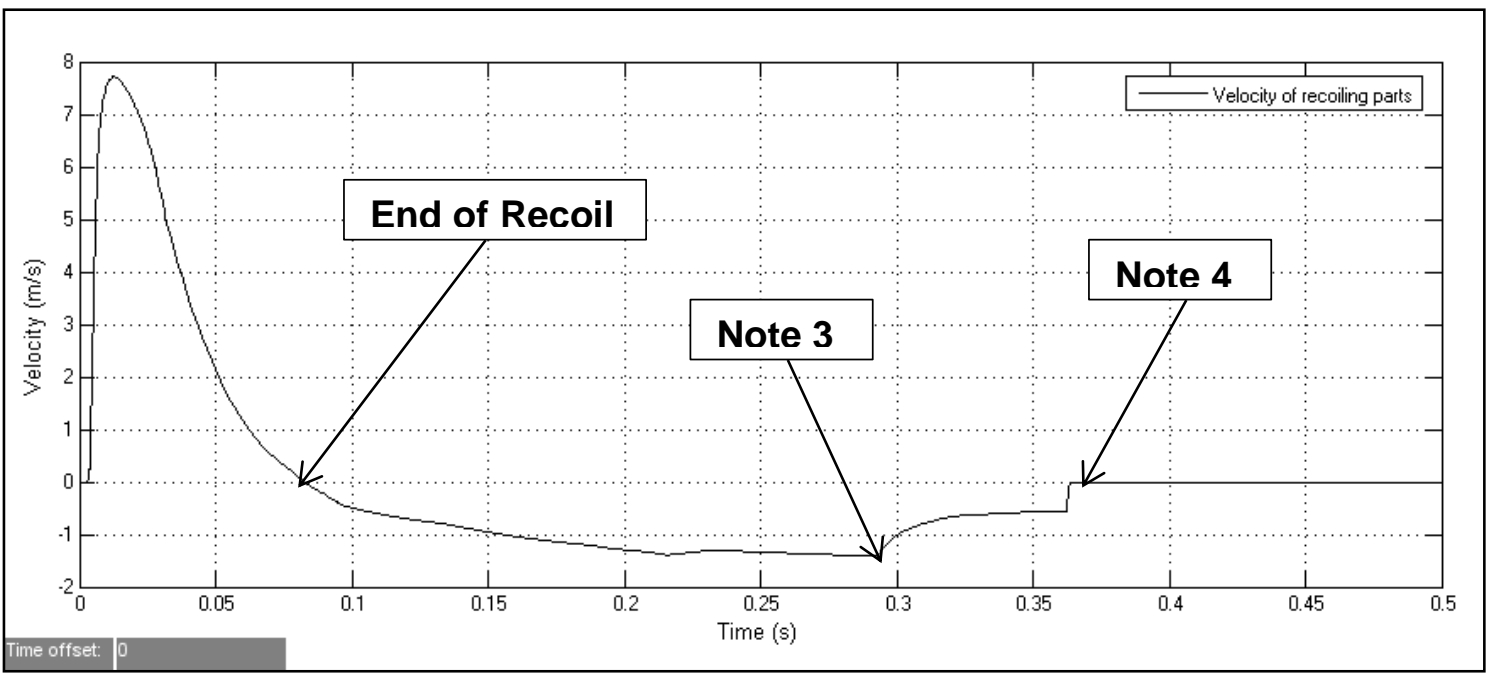

Figure 10. Course of velocity of recoiling parts with time. 


\section{Analysis of Results}

The analysis of results is illustrated as short notes related to the results figures.

\section{Note 1:}

A part of the measured recoil distance between $t=0.1(s)$ up to $t=0.3(s)$ is plotted linearly due to unexpected jump of the gun out the field of view of the high speed camera during measurement.

\section{Note 2:}

The sudden cusps in simulated course for $p_{1}$ are due to the sudden changes in the throttling area. Figure 11 clarifies how the pressure course inside recoil cylinder is affected by changes in recoil cylinder inner profile. These sudden changes cannot be obtained in measurements because the measuring system for pressure inside recoil cylinder is not a zero-order system. Same behavior of the course of the same measured value for another weapon is clear in reference [4].

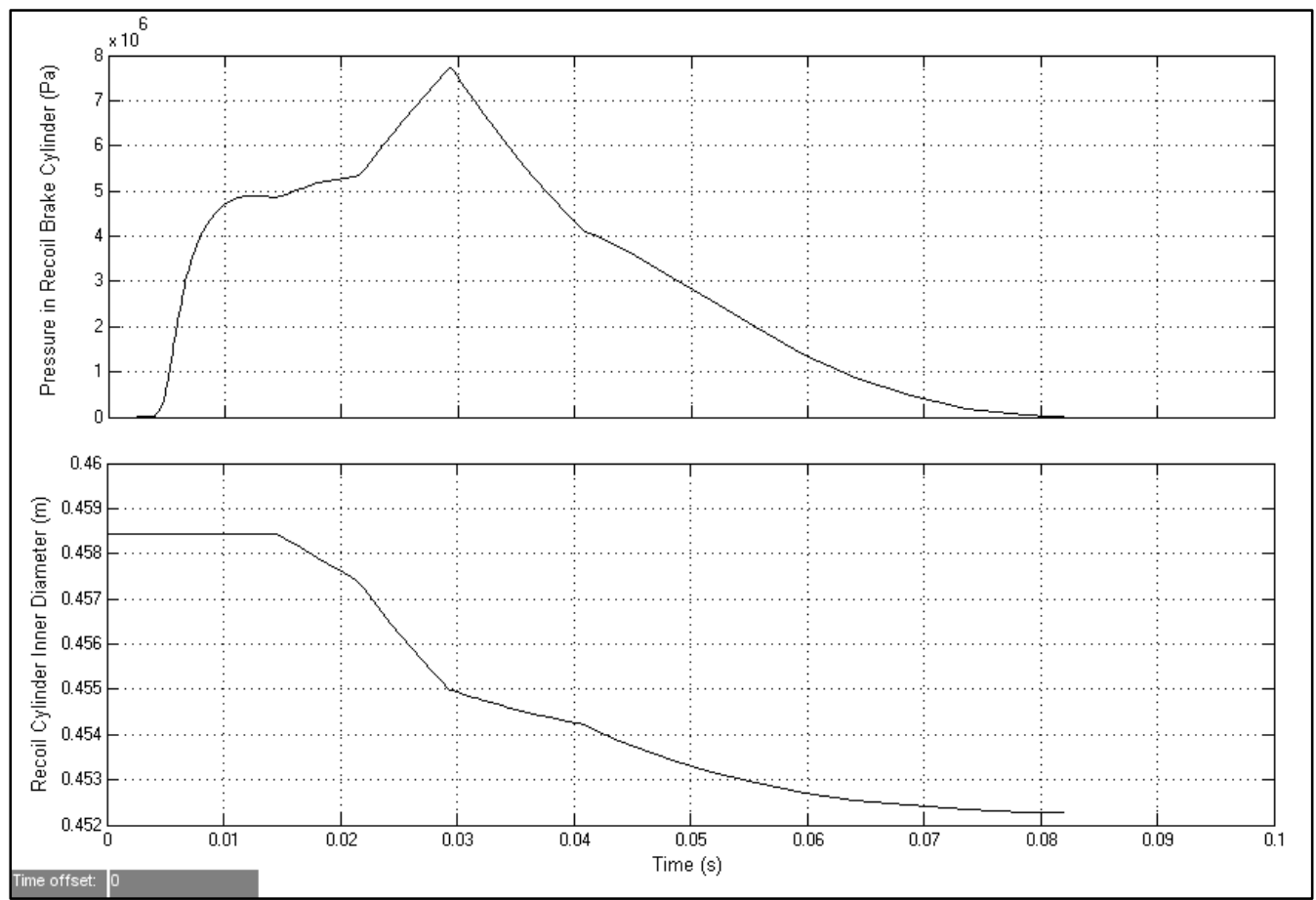

Figure 11. Effect of recoil cylinder inner diameter on simulated $p_{1}$.

\section{Note 3:}

The beginning of cushion effect at the end of counter recoil is clear and causes noticeable change in counter recoil velocity course as shown in Figure 10.

\section{Note 4:}

To accomplish complete counter recoil under all firing conditions, there should be a smooth impact in the barrel front seat. In this simulation, the impact velocity according to simulation does not exceed $0.5(\mathrm{~m} / \mathrm{s})$. 


\section{CONCLUSIONS}

- The established simulation model for gun recoil cycle proved to give results with high confidence for concentric recoil mechanism of tank gun.

- The internal ballistics solution is very useful to get the pressure curve inside the barrel for studying the influence of ammunition type variation on recoil cycle. However, measuring the pressure curve and set it as input for simulation program avoids errors due to ballistics solution.

- Although there is a linearized part in experimental recoil velocity curve with time, there is coincidence between simulated and measured data in total time of recoil cycle and cushion performance. So, this error is not annoying.

- There is some disparity between simulated and measured data for pressure inside the recoil brake cylinder due to many factors mentioned in Ref [3], [4]. So, finding clean techniques for prediction of pressure inside the recoil brake cylinder is worth.

- Estimation of system behavior with variation of some firing parameters is essential. Also, the velocity curve can be set as input to a CFD numerical model and studying performance of flow inside recoil cylinder. Ref [5], [6].

\section{REFERENCES}

[1] A. Hassan, Investigation of the Effect of Constructional Parameters on the Dynamics of Gun Recoil System, MSc Thesis, MTC, Cairo, 2002.

[2] T. Y. Lin, H. C. Ping, T. Y. Yang, C. T. Chan, and C. C. Yang, Dynamic Simulation of the Recoil Mechanism on Artillery Weapons, Journal of ICCES, Vol.11, No.4, pp.115-121, 2009.

[3] Zhang Xiaodong, Zhang Peilin, Fu Jianping, Wang Cheng, Kong Guojie, A Method of Recoil Brake Interior Chamber Pressure Test and Calculation on Gun's Recoiling Impact, The Ninth International Conference on Electronic Measurement \& Instruments, ICEMl'2009.

[4] Zhang Xiaodong, , Zhang Peilin, Fu Jianping, Wang Cheng, Research on According Calculation of Hydraulic Resistance Coefficients for Recoil Brake of Gun Based on Improved Genetic Algorithm, International Conference on Measuring Technology and Mechatronics Automation, 2009.

[5] Lijun Cao, Huibin Hu, Junqi Qin, Condition Detection and Fault Diagnosis System for Recoil System Based on Simulation, The Ninth International Conference on Electronic Measurement \& Instruments, ICEMI'2009.

[6] Guangqun Cao, Faming Zhou, Jiadong Shi, Zhiyin Zeng, and Xiaoke Gao, A numerical method for artillery recoil resistance calculation, Proceedings of ICM, Identification and Control, Wuhan, China, June 24-26, 2012. 